\title{
A New Approximation Algorithm for Fixed Points of Nonexpansive Mappings
}

\author{
Y. $\mathrm{Su}^{1}$ and R.P. Agarwal ${ }^{2}$ \\ ${ }^{1}$ Department of Mathematics, Tianjin Polytechnic University \\ Tianjin 300160, China \\ E-mail(corresp.): suyongfu@gmail.com \\ ${ }^{2}$ Department of Mathematical Science, Florida Institute of Technology \\ Melbourne, FL 32901-6975, USA \\ E-mail: agarwal@fit.edu
}

Received July 12, 2009; revised November 11, 2009; published online April 20, 2010

\begin{abstract}
The aim of this paper is to establish a new approximation algorithm for fixed points of nonexpansive mappings in general Banach spaces and to illustrate some numerical results. The approximation algorithm we shall discuss is $x_{t, n}=(t T)^{n} x_{0}$, where $x_{0} \in D(T)$ is arbitrary, $n$ is a natural number, and $t \in(0,1)$. We shall also provide some numerical error estimates.
\end{abstract}

Keywords: nonexpansive mapping, fixed point, approximation algorithm, numerical error estimation, convergence.

AMS Subject Classification: 47H05; 47H10.

\section{Introduction and Preliminaries}

From the applications point of view the construction of fixed points for nonexpansive mappings is an important topic in the theory of nonlinear analysis. For nonexpansive mappings classical fixed point theorems have been established in [1, 4, 10], whereas Mann [5], Ishikawa [3], and Halpern [2] have analyzed some iterative schemes. Various improvements and extensions of the iterative schemes presented in $[2,3,5]$ have been offered in recent years in $[6,7,8,9,11,13]$. However, although these improvements and extensions advance the theory, they have only limited applicability in applied problems. The purpose of this paper is to study a new approximation algorithm of fixed points for nonexpansive mappings in general Banach spaces and to illustrate its applications in numerical computation. Our approximation scheme is defined by $x_{t, n}=(t T)^{n} x_{0}$, where $x_{0} \in D(T)$ is arbitrary, $n$ is a natural number, and $t \in(0,1)$.

In order to study our new approximation algorithm, the following notation will be useful. 
A mapping $T: K \rightarrow K$, in a Banach space, is said to be almost invariant, if the range $R(T)$ is bounded and there exists some constant $t_{0} \in(0,1)$ such that $t R(T) \subset K$ for all $t \in\left[t_{0}, 1\right]$.

Example 1. Let $E$ be a Banach space and $T$ be a retraction from $K=\{x$ : $\left.\left\|x-x_{0}\right\| \leq 2\right\}$ onto $R(T)=\left\{x:\left\|x-x_{0}\right\| \leq 1 \|\right.$, where $x_{0}$ is a point of $E$. Then for all $\alpha \in[1 / 2,1]$, we have $\alpha R(T) \subset K$, and hence $T$ is almost invariant.

Example 2. Let $E$ be a Banach space, $S(E)$ denote the unit ball of $E$, and let $T: S(E) \rightarrow S(E)$ be a mapping, then $T$ is almost invariant.

\subsection{New approximation algorithm}

Let $E$ be a Banach space, $K$ be a nonempty closed subset of $E$ and $T: K \rightarrow K$ be an almost invariant nonexpansive mapping. For an arbitrary $x_{0} \in K$ we define the sequence $\left\{x_{t, n}\right\}$ by

$$
x_{t, n}=(t T)^{n} x_{0},
$$

where $t \in(0,1)$ and $n$ is a natural number.

Recall that a Banach space $E$ is said to satisfy Opial's condition, if whenever $\left\{x_{n}\right\}$ is a sequence in $E$ which converges weakly to $x$, then

$$
\limsup _{n \rightarrow \infty}\left\|x_{n}-x\right\|<\limsup _{n \rightarrow \infty}\left\|x_{n}-y\right\|, \quad \forall y \in E, \quad y \neq x .
$$

We also recall that a mapping $T$ in a Banach space is said to be demi-closed at zero if for any sequence $\left\{x_{n}\right\}$ which converges weakly to $x^{*}$ and $\left\{T x_{n}\right\}$ converges strongly to zero, $T x^{*}=0$. We shall need the following lemma.

Lemma 1. [12] Let $E$ be a real reflexive Banach space which satisfy Opial's condition. Let $K$ be a nonempty closed convex subset of $E$, and $T: K \rightarrow K$ be a continuous pseudocontractive mapping. Then $(I-T)$ is demi-closed at zero.

Since any nonexpansive mapping is continuous pseudocontractive mapping, the above demi-closed principle also holds for nonexpansive mappings.

\section{Error Estimation for Approximate Fixed Points}

Let $E$ be a real Banach space, $K$ be a closed subset of $E, T: K \rightarrow K$ be an almost invariant nonexpansive mapping and $x_{t, n}$ be the path defined by (1.1). Assume that $t \rightarrow 1$, then there exists some neighbourhood $\left[t_{0}, 1\right)$ such that, for any $t \in\left[t_{0}, 1\right)$, the contraction $t T: K \rightarrow K$ has a unique fixed point $y_{t} \in K$, i.e., $y_{t}=t T y_{t}$. Further, for any given $t \in\left[t_{0}, 1\right)$, by the Banach fixed point theorem, the approximation sequence $\left\{x_{t, n}\right\}$ defined by (1.1) converges strongly to the $y_{t}$, as $n \rightarrow \infty$ (the Picard iterative process). Now from the error estimation formula for the Picard iterative process, we have

$$
\left\|x_{t, n}-y_{t}\right\| \leq \frac{t^{n}}{1-t}\left\|x_{0}-t T x_{0}\right\|
$$


In addition, from $y_{t}=t T y_{t}$, we find

$$
\left\|y_{t}-T y_{t}\right\| \leq(1 / t-1)\left\|y_{t}\right\| .
$$

Combining (2.1) and (2.2), we obtain

$$
\begin{aligned}
\left\|x_{t, n}-T x_{t, n}\right\| & \leq\left\|x_{t, n}-y_{t}\right\|+\left\|y_{t}-T y_{t}\right\|+\left\|T y_{t}-T x_{t, n}\right\| \\
& \leq 2\left\|x_{t, n}-y_{t}\right\|+\left\|y_{t}-T y_{t}\right\| \\
& \leq \frac{2 t^{n}}{1-t}\left\|x_{0}-t T x_{0}\right\|+\left(\frac{1}{t}-1\right)\left\|t T y_{t}\right\| \\
& =\frac{2 t^{n}}{1-t}\left\|x_{0}-t T x_{0}\right\|+(1-t)\left\|T y_{t}\right\| \\
& \leq \frac{2 t^{n}}{1-t}\left\|x_{0}-t T x_{0}\right\|+(1-t) \max _{z \in R(T)}\|z\| \\
& \leq \frac{2 t^{n}}{1-t}\left\|x_{0}-T x_{0}\right\|+2 t^{n}\left\|T x_{0}\right\|+(1-t) \max _{z \in R(T)}\|z\| .
\end{aligned}
$$

Now since $\lim _{t \rightarrow 1, n \rightarrow \infty} \frac{t^{n}}{1-t}=0$, it follows that

$$
\lim _{t \rightarrow 1, n \rightarrow \infty}\left\|x_{t, n}-T x_{t, n}\right\|=0 .
$$

Hence, we have established an approximate fixed point path $\left\{x_{t, n}\right\}=(t T)^{n} x_{0}$ and the error estimation (2.3).

For any given acceptable error $\varepsilon>0$, in the inequality $(2.3)$ we take $t \in(0,1)$ and a natural number $n$ such that $\left\|x_{t, n}-T x_{t, n}\right\| \leq \varepsilon$, where $x_{t, n}=(t T)^{n} x_{0}$. Clearly, to reduce the number of iterations, we must take the least natural numbers $n$ so that the above inequality is satisfied. For this, we consider the following nonlinear optimal problem

$$
\min n=n(t), \quad x_{t, n}=(t T)^{n} x_{0}, \quad\left\|x_{t, n}-T x_{t, n}\right\| \leq \varepsilon, \quad t \in(0,1) .
$$

Next, in the inequality (2.3), we let

$$
A=2\left\|x_{0}-T x_{0}\right\|, \quad B=2\left\|T x_{0}\right\|, \quad C=\max _{z \in R(T)}\|z\|,
$$

so it can be written as

$$
\left\|x_{t, n}-T x_{t, n}\right\| \leq \frac{t^{n}}{1-t} A+t^{n} B+(1-t) C .
$$

Now for any given acceptable error $\varepsilon>0$, we let

$$
\frac{t^{n}}{1-t} A+t^{n} B+(1-t) C \leq \varepsilon,
$$

which is equivalent to

$$
t^{n}\left(\frac{A}{1-t}+B\right) \leq \varepsilon-(1-t) C,
$$


and hence

$$
t^{n} \leq \frac{\varepsilon-(1-t) C}{A /(1-t)+B}=\frac{(1-t) \varepsilon-(1-t)^{2} C}{A+(1-t) B} .
$$

Thus, it follows that

$$
n \geq \frac{\ln \left[\left((1-t) \varepsilon-(1-t)^{2} C\right) /(A+(1-t) B)\right]}{\ln t} .
$$

Next, without any loss of generality, we can assume that

$$
0<\frac{(1-t) \varepsilon-(1-t)^{2} C}{A+(1-t) B}<1
$$

so that $n>0$. Clearly, for any given $\varepsilon>0$, if we take $t \in(0,1)$ and a nature number $n$ such that (2.4) holds, then $\left\|x_{t, n}-T x_{t, n}\right\| \leq \varepsilon$, where $x_{t, n}=(t T)^{n} x_{0}$.

Finally, we define

$$
f_{\varepsilon, A, B, C}(t)=\frac{\ln \left[\left((1-t) \varepsilon-(1-t)^{2} C\right) /(A+(1-t) B)\right]}{\ln t}
$$

and compute the minimum of $f_{\varepsilon, A, B, C}(t)$ in the open interval $(0,1)$, for the given $\varepsilon, A, B, C$. If there exists a $t_{\varepsilon} \in(0,1)$ such that

$$
f_{\varepsilon, A, B, C}\left(t_{\varepsilon}\right)=\min _{t \in(0,1)} f_{\varepsilon, A, B, C}(t),
$$

then we can take $n_{\varepsilon}=\left[f_{\varepsilon, A, B, C}\left(t_{\varepsilon}\right)\right]+1$ and $x_{t_{\varepsilon}, n_{\varepsilon}}=\left(t_{\varepsilon} T\right)^{n_{\varepsilon}} x_{0}$, so that

$$
\left\|x_{\varepsilon, n_{\varepsilon}}-T x_{\varepsilon, n_{\varepsilon}}\right\| \leq \varepsilon .
$$

The above process for the computation of approximate fixed points of the nonexpansive mapping $T$, by the approximate algorithm $x_{t, n}=(t T)^{n} x_{0}$ provides the minimal natural number $n$.

Now we shall give some numerical examples. For simplicity, we shall assume that $A=B=C=1$, and consider the four cases, $\varepsilon=0.5,0.1,0.01,0.001$.

Case 1. Let $\varepsilon=0.5$. In this case, we have

$$
f_{0.5,1,1,1}(t)=\frac{\ln \left[\left(0.5(1-t)-(1-t)^{2}\right) /(1+(1-t))\right]}{\ln t} .
$$

It is clear that, the domain of $f_{0.5,1,1,1}(t)$ must be $0.5<t<1$, and

$$
\lim _{t \rightarrow 0.5^{+}} f_{0.5,1,1,1}(t)=+\infty, \quad \lim _{t \rightarrow 1^{-}} f_{0.5,1,1,1}(t)=+\infty .
$$

We compute the values of $f_{0.5,1,1,1}(t)$ for some $0.5<t<1$ :

$$
\begin{array}{ll}
f_{0.5,1,1,1}(0.63)=7.24898 & f_{0.5,1,1,1}(0.62)=7.13321 \\
f_{0.5,1,1,1}(0.61)=7.03664 & f_{0.5,1,1,1}(0.60)=6.96000 \\
f_{0.5,1,1,1}(0.59)=6.90468 & f_{0.5,1,1,1}(0.58)=6.87296 \\
f_{0.5,1,1,1}(0.57)=6.86848 & f_{0.5,1,1,1}(0.56)=6.89750 \\
f_{0.5,1,1,1}(0.55)=6.96813 & f_{0.5,1,1,1}(0.54)=7.09825
\end{array}
$$


We also compute the minimum of $f_{0.5,1,1,1}(t)$

$$
\min _{0.5<t<1} f_{0.5,1,1,1}(t)=6.86664=f_{0.5,1,1,1}\left(t^{*}\right), \quad t^{*}=0.573433 .
$$

Thus, we can take $x_{t, n}=(0.573433 T)^{7} x_{0}$, as an approximate fixed point of $T$. Clearly, it follows that $\left\|x_{t, n}-T x_{t, n}\right\| \leq 0.5$.

Case 2. Let $\varepsilon=0.1$. In this case, we have

$$
f_{0.1,1,1,1}(t)=\frac{\ln \left[\left(0.1(1-t)-(1-t)^{2}\right) /(1+(1-t))\right]}{\ln t} .
$$

It is clear that, the domain of $f_{0.5,1,1,1}(t)$ must be $0.9<t<1$, and

$$
\lim _{t \rightarrow 0.9^{+}} f_{0.1,1,1,1}(t)=+\infty, \quad \lim _{t \rightarrow 1^{-}} f_{0.1,1,1,1}(t)=+\infty .
$$

We compute the functional values of $f_{0.1,1,1,1}(t)$ for some $0.9<t<1$ in the following:

$$
\begin{array}{ll}
f_{0.1,1,1,1}(0.99)=698.7890 & f_{0.1,1,1,1}(0.95)=117.7590 \\
f_{0.1,1,1,1}(0.94)=98.4326 & f_{0.1,1,1,1}(0.93)=85.8952 \\
f_{0.1,1,1,1}(0.92)=78.1313 & f_{0.1,1,1,1}(0.9109)=75.2484 \\
f_{0.1,1,1,1}(0.91)=75.2756 & f_{0.1,1,1,1}(0.901)=89.3505
\end{array}
$$

We also compute the minimum of $f_{0.1,1,1,1}(t)$

$$
\min _{0.9<t<1} f_{0.1,1,1,1}(t)=75.2475=f_{0.1,1,1,1}\left(t^{*}\right), \quad t^{*}=0.917633 .
$$

Thus, we can take $x_{t, n}=(0.917633 T)^{76} x_{0}$ as an approximate fixed point of $T$. Clearly, it follows that $\left\|x_{t, n}-T x_{t, n}\right\| \leq 0.1$.

Case 3. Let $\varepsilon=0.01$. In this case, we have

$$
f_{0.01,1,1,1}(t)=\frac{\ln \left[\left(0.01(1-t)-(1-t)^{2}\right) /(1+(1-t))\right]}{\ln t} .
$$

It is clear that, the domain of $f_{0.01,1,1,1}(t)$ must be $0.99<t<1$, and

$$
\lim _{t \rightarrow 0.99^{+}} f_{0.01,1,1,1}(t)=+\infty, \quad \lim _{t \rightarrow 1^{-}} f_{0.01,1,1,1}(t)=+\infty .
$$

We compute the functional values of $f_{0.01,1,1,1}(t)$ for some $0.99<t<1$ in the following:

$$
\begin{array}{ll}
f_{0.01,1,1,1}(0.9920)=1375.83 & f_{0.01,1,1,1}(0.9912)=1297.35 \\
f_{0.01,1,1,1}(0.9911)=1291.19 & f_{0.01,1,1,1}(0.9910)=1286.09 \\
f_{0.01,1,1,1}(0.9909)=1282.22 & f_{0.01,1,1,1}(0.9908)=1279.79 \\
f_{0.01,1,1,1}(0.9907)=1279.11 & f_{0.01,1,1,1}(0.9906)=1280.64 \\
f_{0.01,1,1,1}(0.9905)=1285.10 & f_{0.01,1,1,1}(0.9904)=1293.71
\end{array}
$$

We also compute the minimum of $f_{0.01,1,1,1}(t)$

$$
\min _{0.99<t<1} f_{0.01,1,1,1}(t)=1279.09=f_{0.01,1,1,1}\left(t^{*}\right), \quad t^{*}=0.99071541 .
$$

Thus, we can take $x_{t, n}=(0.99071541 T)^{1280} x_{0}$ as an approximate fixed point of $T$. Clearly, it follows that $\left\|x_{t, n}-T x_{t, n}\right\| \leq 0.01$. 
Case 4. Let $\varepsilon=0.001$. In this case, we have

$$
f_{0.001,1,1,1}(t)=\frac{\ln \left[\left(0.001(1-t)-(1-t)^{2}\right) /(1+(1-t))\right]}{\ln t} .
$$

It is clear that, the domain of $f_{0.001,1,1,1}(t)$ must be $0.999<t<1$, and

$$
\lim _{t \rightarrow 0.999^{+}} f_{0.001,1,1,1}(t)=+\infty, \quad \lim _{t \rightarrow 1^{-}} f_{0.001,1,1,1}(t)=+\infty .
$$

We compute the functional values of $f_{0.001,1,1,1}(t)$ for some $0.999<t<1$ in the following:

$$
\begin{array}{ll}
f_{0.001,1,1,1}(0.9991)=18018.90 & f_{0.001,1,1,1}(0.99909)=17924.50 \\
f_{0.001,1,1,1}(0.99908)=17845.60 & f_{0.001,1,1,1}(0.99907)=17785.60 \\
f_{0.001,1,1,1}(0.99906)=17748.80 & f_{0.001,1,1,1}(0.99905)=17742.60 \\
f_{0.001,1,1,1}(0.99904)=17779.10 & f_{0.001,1,1,1}(0.99903)=17881.50 \\
f_{0.001,1,1,1}(0.99902)=18102.10 & f_{0.001,1,1,1}(0.99901)=18608.70
\end{array}
$$

We also compute the minimum of $f_{0.001,1,1,1}(t)$

$$
\min _{0.999<t<1} f_{0.001,1,1,1}(t)=17740.70=f_{0.001,1,1,1}\left(t^{*}\right), \quad t^{*}=0.999053156 .
$$

Thus, we can take $x_{t, n}=(0.999053156 T)^{17741} x_{0}$ as an approximate fixed point of $T$. Clearly, it follows that $\left\|x_{t, n}-T x_{t, n}\right\| \leq 0.001$.

\section{Some Approximation Fixed Point Sequences and Con- vergence}

In particular, let $t=\alpha_{n} \rightarrow 1$, as $n \rightarrow \infty$, be a real sequence in $(0,1)$ such that $\lim _{n \rightarrow \infty} \frac{\alpha_{n}^{n}}{1-\alpha_{n}}=0$, then the approximation algorithm (1.1) takes the following special form

$$
x_{n}=\left(\alpha_{n} T\right)^{n} x_{0} .
$$

Clearly, from (2.3) it follows that

$$
\lim _{n \rightarrow \infty}\left\|x_{n}-T x_{n}\right\|=0
$$

Example 3. Let $\alpha_{n}=\sqrt{n} /(\sqrt{n}+1)$. Then, it is easy to show that

$$
\frac{\alpha_{n}^{n}}{1-\alpha_{n}}=\frac{\left(\frac{\sqrt{n}}{\sqrt{n}+1}\right)^{n}}{1-\frac{\sqrt{n}}{\sqrt{n}+1}}=\frac{\left(\frac{\sqrt{n}}{\sqrt{n}+1}\right)^{n}}{\frac{1}{\sqrt{n}+1}} \rightarrow 0
$$

as $n \rightarrow \infty$. In this case, the approximation algorithm (1.1) simply takes the following form

$$
x_{n}=\left(\frac{\sqrt{n}}{\sqrt{n}+1} T\right)^{n} x_{0} .
$$


Theorem 1. Let $E$ be a real reflexive Banach space which satisfies Opial's condition, $K$ be a closed convex subset of $E$, and $T: K \rightarrow K$ be an almost invariant nonexpansive mapping. Then the fixed points set $F(T)$ is nonempty. Moreover let $\lim _{n \rightarrow \infty} \frac{\alpha_{n}^{n}}{1-\alpha_{n}}=0$. Then for any initial $x_{0} \in K, w_{w}\left(x_{n}\right) \subset F(T)$, where $w_{w}\left(x_{n}\right)$ denote the weak limit set of $\left\{x_{n}\right\}$ defined by (3.1).

Proof. From (3.2) we have $\left\|x_{n}-T x_{n}\right\| \rightarrow 0$ as $n \rightarrow \infty$. This together with the reflexivity of Banach space $E$, and Lemma 1, implies that, for any subsequence $\left\{x_{n_{k}}\right\}$ of $\left\{x_{n}\right\}$, there must exist a subsequence $\left\{x_{n_{k_{i}}}\right\}$ of $\left\{x_{n_{k}}\right\}$ which converges weakly to a fixed point $x^{*}$ of $T$. This completes the proof.

Example 4. From Example 3, it follows that the sequence $\left\{x_{n}\right\}$ defined by

$$
x_{n}=\left(\frac{n}{n+1} T\right)^{n^{2}} x_{0}
$$

is also an approximation fixed point sequence. The numerical error estimates of (3.3) and (3.4) can be computed as in Section 2.

Theorem 2. Let $E$ be a real reflexive Banach space which satisfy Opial's condition, $K$ be a closed convex subset of $E$, and $T: K \rightarrow K$ be an almost invariant nonexpansive mapping. Then the fixed points set $F(T)$ is nonempty and for any initial $x_{0} \in K, w_{w}\left(x_{n}\right) \subset F(T)$, where $w_{w}\left(x_{n}\right)$ denote the weak limit set of $\left\{x_{n}\right\}$ defined by (3.4).

\section{References}

[1] K. Goebel and W. Kirk. A fixed point theorem for asymptotically nonexpansive mappings. Proc. Amer. Math. Soc., 35:171-174, 1972. Doi:10.2307/2038462.

[2] B. Halpern. Fixed points of nonexpansive maps. Bull. Amer. Math. Soc., 73:957961, 1976. Doi:10.1090/S0002-9904-1967-11864-0.

[3] S. Ishikawa. Fixed point by new iteration method. Proc. Amer. Math. Soc., 44:147-150, 1974. Doi:10.2307/2039245.

[4] T.H. Kim and H.K. Xu. Remarks on asymptotically nonexpansive mappings. Nonlinear Analysis, 41:405-415, 2000. Doi:10.1016/S0362-546X(98)00284-3.

[5] W. Mann. Mean value methods in iteration. Proc. Amer. Math. Soc., 4:506-510, 1953. Doi: $10.2307 / 2032162$.

[6] A. Moudafi. Viscosity approximation methods for fixed point problems. J. Math. Anal. Appl., 241:46-55, 2000. Doi:10.1006/jmaa.1999.6615.

[7] N. Nakajo and W. Takahashi. Strong convergence theorems for nonexpansive mappings and nonexpansive semi-groups. J. Math. Anal. Appl., 297:372-379, 2003. Doi:10.1016/S0022-247X(02)00458-4.

[8] M. Noor and Z. Huang. Wiener-Hopf equation technique for variational inequalities and nonexpansive mappings. Appl. Math. Comput., 2007.

Doi:10.1016/j.amc.2007.02.117.

[9] Y. Su and X. Qin. General iteration algorithm and convergence rate optimal model for common fixed points of nonexpansive mappings. Appl. Math. Comput., 186:271-278, 2007. Doi:10.1016/j.amc.2006.07.101. 
[10] W. Takahashi. Nonlinear Functional Analysis, Fixed Point Theory and Applications. Yokohama Publishers, 2000.

[11] H. Xu and M. Ori. An implicit iteration process for nonexpansive mappings. $\mathrm{Nu}$ mer. Funct. Anal. Optimize., 22:767-773, 2001. Doi:10.1081/NFA-100105317.

[12] H. Zhou. Convergence theorems of common fixed points for a finite family of Lipschitz pseudocontractions in Banach spaces. Nonlinear Analysis, 2007. Doi:10.1016/j.na.2007.02.041.

[13] H. Zhou and S. Chang. Convergence of implicit iterative process for a finite family of asymptotically nonexpansive mappings in Banach spaces. Numer. Funct. Anal. Optimize., 23:911-921, 2002. Doi:10.1081/NFA-120016276. 